J. Lake Sci. (湖泊科学), 2012, 24(5): 663-666

http: //www.jlakes.org. E-mail : jlakes@niglas.ac.cn

(C) 2012 by Journal of Lake Sciences

\title{
河道入湖污染物量计算精度分析”
}

马 倩 ${ }^{1}$, 冯 昕 ${ }^{2}$

(1: 江苏省水文水资源勘测局,南京 210029)

(2: 北京大学地球与空间科学院,北京 100871 )

摘 要: 人湖污染物量计算精度的高低决定人湖污染物量分析结果的客观性和准确性. 本文以太湖为例, 分析 2010 年环 太湖河道人湖污染物量、时空分布情况及多年环太湖河道人湖染污物量变化情势. 在此基础上,使用现在已有监测条件 分析时段内每日水量水质监测数据计算逐日人湖污染物量时段累积值, 并以此作为现有分析计算河道人湖污染物量的 最高计算精度值. 通过设定不同监测方案、采用不同计算方法分析河道人湖污染物量及其计算精度,认为现有分析计算 的河道人湖污染物量已是每日河道水量水质同步监测下河道人湖污染物量的 $80 \%$ 左右. 针对河道人湖污染物量计算精 度的分析可为制定科学的河道人湖污染量监测方案、提高河道人湖污染物量计算精度提供技术支撑.

关键词: 污染物量;太湖;计算精度;河道

\section{Calculation precision of pollutant load of rivers}

\section{MA Qian ${ }^{1} \&$ FENG Xin ${ }^{2}$}

( 1 : Jiangsu Hydrology and Water Resources Investigation Bureau, Nanjing 210029, P. R. China)

(2: Institute of Remote Sensing and GIS, Peking University, Beijing 100871, P. R. China)

Abstract: The objectivity and accuracy of the analysis results of pollutant load into the Lake Taihu are controlled by the calculation precision. The paper takes Lake Taihu as an example, analyses not only pollutant load of rivers into Lake Taihu in 2010, but also the temporal and spatial distributions and variation of the pollutant load. The daily monitoring data of water yield and water quality under the existing monitoring network are used to calculate pollutant load into the Lake Taihu in a daily accumulation period, which is assumed the highest calculation precision for existing analysis and calculation of pollutant load of rivers into the Lake Taihu. With different monitoring schemes and different calculation methods, the study analysed pollutant load of rivers into the Lake Taihu and the calculation precision achieved. It is found that pollutant load of rivers into the lake analysed and calculated nowadays accounts for 80 percents of which monitored everyday simultaneously. Technical support is provided by analysing the calculation precision of pollutant load of rivers into the Lake Taihu to make scientific monitoring schemes and to improve the computation precision of the pollutant load of rivers into the Lake Taihu.

Keywords: Pollutant load; Lake Taihu; calculation precision; rivers into the lake

人湖污染物量计算精度分析是评价我们分析计算人湖污染物量可靠性、真实性的手段之一. 有关人湖 污染物量计算方法, 国内外研究成果归纳起来, 主要是考虑人湖污染物量随时间变化, 采用在分析时段内先 将计算时段的水量值与水质浓度值求积再求和 ${ }^{[1]}$ 或是分别计算分析时段内的水量平均与水质平均浓度值 之和再求积 ${ }^{[2]}$ 的方法. 由于河道断面水质监测频次低, 国内外对河道入湖污染物量计算精度的分析、评价与 研究较少, 对计算所得的河道人湖污染物量的准确性缺乏较为一致的认识. 本文以太湖为例, 分析现有监测 状况下 2010 年江苏省河道人太湖污染物量及其变化情势, 并根据已掌握的人湖河道一定时段内每日水量 水质同步监测成果, 针对不同的计算方法与现有常见的监测方案, 分析人湖污染物量, 评价人湖污染物量的 计算精度,为有效掌控河道的人湖污染物量、提出限制排污总量意见提供技术支撑与决策依据.

* 2011-11-19 收稿;2012-03-20 收修改稿. 马倩,女, 1961 年生, 研究员级高级工程师; E-mail : maq210029@yahoo. com. cn. 


\section{1 入太湖污染物量现状分析}

2010 年江苏省环太湖河道人湖水量、氨氮、总磷、总氮和高锰酸盐指数 $\left(\mathrm{COD}_{\mathrm{Mn}}\right)$ 分别为 $93.3 \times 10^{8} \mathrm{~m}^{3}$ 、 $1.48 \times 10^{4} \mathrm{t} 、 0.21 \times 10^{4} \mathrm{t} 、 4.13 \times 10^{4} \mathrm{t} 、 24.7 \times 10^{4} \mathrm{t}$, 分别为多年平均的 $1.272 、 0.778 、 1.244 、 1.119$ 和 1.276 倍. 消除人湖水量的影响, 2010 年总磷、 $\mathrm{COD}_{\mathrm{Mn}}$ 的人湖水质指标浓度值与多年平均值相当, 氨氮、总氮的人湖 水质指标浓度值比多年平均值低.

2010 年汛期 (5-9 月) 江苏省环太湖河道人湖水量占总人湖水量的 $48.7 \%, 7 、 9 、 10$ 月人湖水量占年总 人湖水量比例超过 $10 \%$; 汗期氨氮人湖污染物量占全年的 $35.3 \%, 2 、 7 、 12$ 月人湖污染物量占全年的比例超 过 $10 \%$; 汛期总磷人湖污染物量占全年的 $44.2 \%, 2 、 7 、 9 、 10$ 月人湖污染物量占全年的比例超过 $10 \%$; 汛期 总氮人湖污染物量占全年的 $37.7 \%, 2-4$ 月、7 月人湖污染物量占全年的比例超过 $10 \%$; 汛期 $\mathrm{COD}_{\mathrm{Mn}}$ 人湖污 染物量占全年的 50.7\%，7、9 月人湖污染物量占全年的比例超过 $10 \%$.

2010 年江苏省太湖湖西地区河道的人湖水量及人湖污染物量占江苏省河道总人湖水量及总人湖污染 物量的 70\% 75\%、武澄锡虞地区占 $25 \%$ 左右、阳澄淀泖区占 $1 \%$ ～2\%、浦南区占不足 $1 \%$; 消除人湖水量的 影响,湖西区人湖氨氮、总磷、总氮、COD ${ }_{\mathrm{Mn}}$ 浓度最高, 武澄锡虞区的人湖总磷、总氮浓度较高 $($ 表 1$)$.

表 12010 年江苏省太湖流域分区河道人湖水量及人湖污染物量比例

Tab. 1 Ratios of water yield and pollutant load of rivers into Lake Taihu of the sub-region from the basin in Jiangsu Province in 2010

\begin{tabular}{cccccc}
\hline \multirow{2}{*}{ 地区 } & \multirow{2}{*}{ 人湖水量比例 $/ \%$} & \multicolumn{4}{c}{ 人湖污染物量比例 $/ \%$} \\
\cline { 3 - 6 } & & 氨氮 & 总磷 & 总氮 & $\mathrm{COD}_{\mathrm{Mn}}$ \\
\hline 湖西 & 70.1 & 74.9 & 71.4 & 72.4 & 74.3 \\
武澄锡虞 & 27.4 & 23.9 & 27.7 & 26.1 & 23.8 \\
阳澄淀泖 & 2.40 & 1.18 & 0.859 & 1.43 & 1.82 \\
浦南区 & 0.082 & 0.019 & 0.018 & 0.029 & 0.058 \\
\hline
\end{tabular}

\section{2 入太湖污染物量情势分析}

江苏省环太湖河道 2003-2010 年氨氮的人湖污染物量总体呈下降趋势; 2003-2006 年总磷的人湖污 染物量呈下降趋势, 2006-2010 年有所增加; 2003-2008 年总氮呈下降趋势, 2008-2010 年有所增加; $2003-2007$ 年 $\mathrm{COD}_{\mathrm{Mn}}$ 呈下降趋势, 2007-2010 年有所增加. 若不考虑人湖水量变化的影响, 2006-2007 年以 来, 江苏省环太湖河道氨氮的人湖污染物量减少明显, 总氮与总磷的量减少次之, $\mathrm{COD}_{\mathrm{Mn}}$ 的量变化不大 (图 1).

\section{3 入湖污染物量计算精度分析}

1998-2003 年江苏省水质断面监测频次 4 6 次/a,2004 年后随着全省主要水功能区监测工作的开展, 水质监测频次提高为 $6 \sim 12$ 次/a, 受 2007 年无锡太湖贡湖发生供水危机影响, 江苏省水文水资源勘测局对 太湖流域人湖河道的水量水质同步监测采用不同的监测频次, 针对现有的监测条件, 我们假定, 选择一定时 期内的一些有每日水量水质同步监测的人湖河道断面监测成果, 以月为时段长, 将逐日监测的水量值与水 质浓度值之积求和作为月人湖污染物量, 作为当前监测条件下计算人湖污染物量的最高精度值, 同时与现 行的常见监测频次,不同的人湖污染物量计算方法进行比较,分析计算精度的差异.

\section{1 计算河道断面和计算时段}

根据现有监测资料, 选择了太湖湖西主要人湖河道烧香港的棉堤桥、陈东港的陈东港桥、太滆运河的黄 埝桥、漕桥河的漕桥共 4 个监测断面,选取的计算时段为: 2007 年 8-9 月、2008 年 4-10月、2009 年 510 月.

\section{2 计算方案设置}

计算方案主要针对常见的几种监测频次以及不同的计算方法而设置, 目前江苏省水文水资源勘测局在 

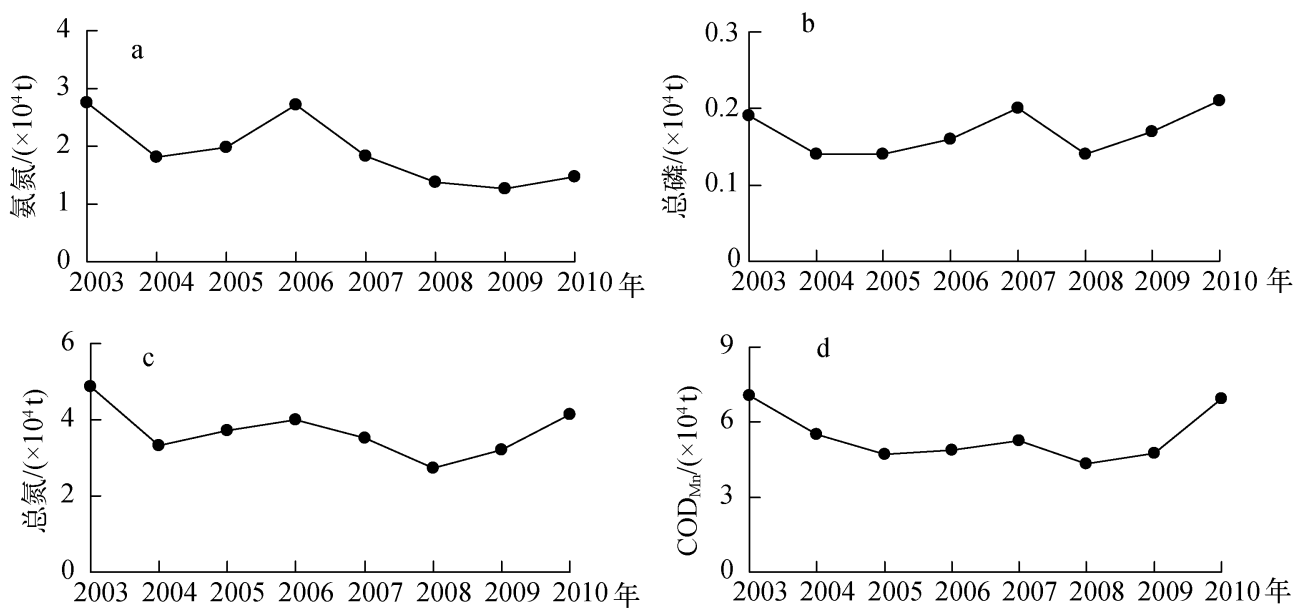

图 1 江苏省环太湖河道人湖污染物量变化(2003-2010 年)

Fig. 1 Variation of pollutant load of rivers into Lake Taihu in Jiangsu Province, 2003 - 2010

太湖地区常用的监测频次为: 每日水量水质同步监测 (主要是在护水控藻、调水引流监测期实施, 对环太湖 主要的人湖河道进行监测,近年来相对时段较长些,监测断面较多)、每月水量水质同步监测 2 次 (每月的 8 日和 23 日监测, 主要在对环太湖出入湖河道进行巡测中实施)、每月逢 $0 、 5$ 日水量水质同步监测 (主要是在 每年的非调水引流期和调水引流稳定期进行实施) ; 计算人湖污染物量的方法最主要的差异在于是先计算 逐日的人湖污染物量再逐日求和作为时段内的人湖污染物量还是先分别计算时段内水量水质监测成果的 平均值再求积作为时段内的人湖污染物量,这两种方法计算成果考虑的侧重面不同,用水量水质同步监测 成果值求积再求和计算的人湖污染物量侧重考虑了人湖污染物量的时程变化过程,突出了个别大的或小的 人湖污染物的作用,而先分别计算水量水质之和再求积计算的人湖污染物量均化了时段内人湖污染物量的 变化. 现设置了 8 种计算人湖污染物量的方案,具体见表 2.

表 2 人湖污染物量计算方案

Tab. 2 Calculation schemes of pollutant load into Lake Taihu

\begin{tabular}{|c|c|c|c|}
\hline 方案 & 水量监测频次/(次/月) & 水质监测频次/ (次/月) & 计算方法 \\
\hline 方案 1 & 逐日监测 & 逐日监测 & 逐日水量水质监测成果积之和 \\
\hline 方案 2 & 逐日监测 & 逐日监测 & 逐日水量水质监测成果和之积 \\
\hline 方案 3 & 逐日监测 & $2(8$ 日、23 日) & 水量水质监测月均值之积按月的日数扩放 \\
\hline 方案 4 & 逐日监测 & $6($ 逢 0.5 日) & 水量水质监测月均值之积按月的日数扩放 \\
\hline 方案 5 & 2 ( 8 日、23 日) & $2(8$ 日、23 日) & 水量水质监测月均值之积按月的日数扩放 \\
\hline 方案 6 & 6(逢0、5 日) & 6(逢0、5 日) & 水量水质监测月均值之积按月的日数扩放 \\
\hline 方案 7 & $2(8$ 日、23日) & $2(8$ 日、23 日) & 次水量水质监测成果积之和按月的日数扩放 \\
\hline 方案 8 & 6 (逢 $0 、 5$ 日) & 6(逢 $0 、 5$ 日) & 次水量水质监测成果积之和按月的日数扩放 \\
\hline
\end{tabular}

\section{3 计算方案比较}

以已有时段内每日水量水质监测成果及逐日累积的时段内人湖污染物量计算成果为现有分析计算河 道人湖污染物量的最高计算精度, 也就是计算方案 1 作为当前河道人湖污染物量计算成果精度最高的,逐 月分析其它各计算方案中不同人湖污染物量与方案 1 的计算成果值的相对误差在 $\pm 5 \% 、 \pm 10 \%$ 之内所占 的综合比值 (表 3 ).

分析比较成果表可以得出:

1) 水量监测的准确与否对计算人湖污染物量的精度非常关键. 方案 $2 、 3 、 4$ 水量采用逐日监测成果,计 
表 3 其它计算方案与方案 1 成果比较

Tab. 3 Comparison between

scheme No. 1 and other schemes

\begin{tabular}{ccc}
\hline \multirow{2}{*}{ 方案 } & \multicolumn{2}{c}{ 误差范围 } \\
\cline { 2 - 3 } & $\pm 5 \%$ & $\pm 10 \%$ \\
\hline 方案 2 & 74.6 & 85.8 \\
方案 3 & 34.2 & 57.9 \\
方案 4 & 44.2 & 71.7 \\
方案 5 & 16.3 & 33.8 \\
方案 6 & 27.1 & 50.8 \\
方案 7 & 15.4 & 35.4 \\
方案 8 & 24.6 & 49.2 \\
\hline
\end{tabular}

算的人湖污染物量成果相对方案 1 误差在 $\pm 10 \%$ 之内的比例在 $55 \% \sim 86 \%$ 之间; 方案 5、6、7、8 水量采用月内定期水量监测成果, 计 算的人湖污染物量成果相对方案 1 误差在 $\pm 10 \%$ 之内的比例在 $33 \% \sim 51 \%$ 之间. 方案 $2 、 3 、 4$ 计算成果精度要比方案 $5 、 6 、 7 、 8$ 高 $20 \% \sim 35 \%$.

2) 水量水质监测的频次越高,计算人湖污染物量精度也就越 高. 方案 3 与方案 4 比较, 水量监测频次相同, 水质监测方案 4 是 6 次/月、方案 3 是 2 次/月,方案 4 比方案 3 计算的人湖污染物量成果 误差在 $\pm 10 \%$ 之内的比例高近 $15 \%$; 方案 $6 、 8$ 水量水质监测 6 次/月、方案 $5 、 7$ 仅 2 次/月,方案 $6 、 8$ 比方案 $5 、 7$ 计算的人湖污染物 量成果误差在 $\pm 10 \%$ 之内的比例也高了近 $15 \%$.

3 ) 水量水质同步监测频次不高时,采用采用水量水质监测成果 积之和与水量水质监测成果和之积, 对计算人湖污染物量差异不

大. 方案 5 与方案 7 、方案 6 与方案 8 水量水质监测频次相同,仅计算方法不同,计算的人湖污染物量成果误 差在 $\pm 10 \%$ 之内的比例在 $2 \%$ 以下.

通过对不同计算人湖污染物量方案成果精度的分析,鉴于江苏省目前对太湖环湖河道建立了比较完善 的由国家基本水文站、巡测基点站、巡测站组成的水量水质同步监测站网,监测也由原来主要在汗期进行的 $20 \sim 22$ 次/ $\mathrm{a}$ 的水量监测, 提高为 2 次/月、汛期加密监测, 且对主要的人湖河道在每年的 4 月 10 日-10 月 20 日进行每日水量水质同步监测, 综合以上人湖污染物量精度分析成果, 我们认为现在分析计算的江苏省河 道人湖污染物量已是每日河道水量水质同步监测并计算河道人湖污染物量的 $80 \%$ 左右.

\section{4 归纳与展望}

太湖水体的纳污能力总磷 $0.051 \times 10^{4} \mathrm{t}$ 、总氮 $0.851 \times 10^{4} \mathrm{t} 、 \mathrm{COD}_{\text {Мп }} 3.437 \times 10^{4} \mathrm{t}$, 江苏省内仅河道进人太 湖的污染物量已远远超出湖泊水体的自净能力,且没有明显减少的趋势 ${ }^{[34]}$.

对太湖河道人湖水量的计算方法,目前仅是使用一些常规的、传统的水文计算方法,随着计算机技术飞 速发展, 以后可以尝试引进、借用或创建一些适应平原水网区水文分析计算的模型, 提高对入湖污染物量分 析计算的精度.

怎样的监测方式、监测频次和计算方法能够得到具有较高精度的人湖污染物量成果有待进一步研究. 监测技术水平的提高、监测仪器设备的不断改善,特别是在出人湖主要河道上建设的实时在线自动水量水 质监测站,是提高人湖污染物量计算精度的有效保障.

\section{5 参考文献}

[1] 马 倩, 刘俊杰, 高明远. 江苏省人太湖污染量分析 (1998-2007 年). 湖泊科学, 2010,22 (1):29-34.

[2] 郑一,王学军,江耀慈等. 环太湖河道水质分析与人湖污染物负荷量估算. 地理学与国土研究, $2001,17(1): 40-44$.

[ 3 ] 许 梅, 任瑞丽, 刘茂松. 太湖人湖河流水质指标的年变化规律. 南京林业大学学报: 自然科学版, 2007,31 (6) : $121-124$.

[4] 秦伯强. 太湖生态与环境若干问题的研究进展及其展望. 湖泊科学,2009,21 (4):445-455. 